The Astrophysical Journal, 522:L65-L68, 1999 September 1

(C) 1999. The American Astronomical Society. All rights reserved. Printed in U.S.A.

\title{
DISCOVERY OF FOUR FIELD METHANE (T-TYPE) DWARFS WITH THE TWO MICRON ALL-SKY SURVEY ${ }^{1}$
}

\author{
Adam J. Burgasser, ${ }^{2}$ J. Davy Kirkpatrick, ${ }^{3}$ Michael E. Brown, ${ }^{4,5}$ I. Neill Reid, \\ John E. Gizis, ${ }^{7}$ Conard C. Dahn, ${ }^{8}$ David G. Monet,${ }^{8}$ Charles A. Beichman, ${ }^{9}$ \\ James Liebert, ${ }^{10}$ Roc M. Cutri, ${ }^{3}$ and Michael F. Skrutskie ${ }^{7}$ \\ Received 1999 June 10; accepted 1999 July 1; published 1999 August 3
}

\begin{abstract}
We report the discovery of four field methane ("T"-type) brown dwarfs using Two Micron All-Sky Survey (2MASS) data. One additional methane dwarf, previously discovered by the Sloan Digital Sky Survey, was also identified. Near-infrared spectra clearly show the 1.6 and $2.2 \mu \mathrm{m} \mathrm{CH}_{4}$ absorption bands characteristic of objects with $T_{\text {eff }} \lesssim 1300 \mathrm{~K}$ as well as broadened $\mathrm{H}_{2} \mathrm{O}$ bands at 1.4 and $1.9 \mu \mathrm{m}$. Comparing the spectra of these objects with that of Gl 229B, we propose that all new 2MASS T dwarfs are warmer than $950 \mathrm{~K}$, in order from warmest to coolest: 2MASS J1217-03, 2MASS J1225-27, 2MASS J1047+21, and 2MASS J1237+65. Based on this preliminary sample, we find a warm $\mathrm{T}$ dwarf surface density of $0.0022 \mathrm{~T}$ dwarfs $\mathrm{deg}^{-2}$, or $\sim 90 \mathrm{warm} \mathrm{T}$ dwarfs over the whole sky detectable to $J<16$. The resulting space density upper limit, $0.01 \mathrm{~T} \mathrm{dwarfs} \mathrm{pc}^{-3}$, is comparable to that of the first L dwarf sample from Kirkpatrick et al.
\end{abstract}

Subject headings: infrared: stars — stars: fundamental parameters stars: individual (2MASSI J1047539+212423, 2MASSW J1217111-031113, 2MASSW J1225543-273947, 2MASSW J1237392+652615, 2MASSW J1346464-003150) stars: low-mass, brown dwarfs

\section{INTRODUCTION}

Searches for brown dwarfs are meeting with increasing success in recent years. Proper motion surveys (Ruiz, Leggett, \& Allard 1997), surveys of young clusters (Stauffer et al. 1989; Rebolo, Zapatero-Osorio, \& Martín 1995), companion searches (Nakajima et al. 1995), radial velocity measurements (Mayor \& Queloz 1995; Marcy \& Butler 1995), and all-sky near-infrared and deep optical surveys (Delfosse et al. 1997; Kirkpatrick et al. 1999, hereafter K99) have identified an evergrowing number of confirmed substellar objects. Until recently, G1 229B (Nakajima et al. 1995) stood out among those objects as the only known brown dwarf sufficiently cool $\left(T_{\text {eff }}=\right.$ $960 \pm 70 \mathrm{~K}$; Marley et al. 1996) to show $\mathrm{CH}_{4}$ absorption bands at 1.6 and $2.2 \mu \mathrm{m}$. Analysis of preliminary data from the Sloan Digital Sky Survey (SDSS; York et al. 1999) has led to the discovery of the first field counterparts of Gl 229B (Strauss et al. 1999; Tsvetanov et al. 1999).

In this Letter, we report the discovery of four additional spectroscopically similar dwarfs, which we designate as spec-

\footnotetext{
${ }^{1}$ Portions of the data presented herein were obtained at the W. M. Keck Observatory, which is operated as a scientific partnership among the California Institute of Technology, the University of California, and the National Aeronautics and Space Administration. The Observatory was made possible by generous financial support of the W. M. Keck Foundation.

${ }^{2}$ Division of Physics, M/S 103-33, California Institute of Technology, Pasadena, CA 91125; diver@cco.caltech.edu.

${ }^{3}$ Infrared Processing and Analysis Center, M/S 100-22, California Institute of Technology, Pasadena, CA 91125.

${ }^{4}$ Division of Geological and Planetary Sciences, M/S 105-21, California Institute of Technology, Pasadena, CA 91125.

${ }^{5}$ Alfred P. Sloan Research Fellow.

${ }^{6}$ Department of Physics and Astronomy, University of Pennsylvania, 209 South 33d Street, Philadelphia, PA 19104-6396.

${ }^{7}$ Five College Astronomy Department, Department of Physics and Astronomy, University of Massachusetts, Amherst, MA 01003.

${ }^{8}$ US Naval Observatory, P.O. Box 1149, Flagstaff, AZ 86002.

${ }^{9}$ Jet Propulsion Laboratory, M/S 180-703, 4800 Oak Grove Drive, Pasadena, CA 91109.

${ }^{10}$ Steward Observatory, University of Arizona, Tuscon, AZ 85721.
}

tral class "T"11 (K99) based on data from the Two Micron AllSky Survey (2MASS; Skrutskie et al. 1997), and the recovery of a $\mathrm{T}$ dwarf identified by SDSS. A brief summary of the selection process and initial follow-up of 2MASS T candidates is discussed in $\S 2$; near-infrared spectroscopy of the confirmed $\mathrm{T}$ dwarfs is discussed in $\S 3$; and a preliminary sequence and estimates of $T_{\text {eff }}$ and the T dwarf space density are discussed in $\S 4$. Results are summarized in $\S 5$.

\section{CANDIDATE SELECTION}

\subsection{Search Criteria}

Candidates were culled from two separate 2MASS data samples. The first (sample A) was taken from the 2MASS Spring 1999 Data Release, ${ }^{12} 2483$ deg $^{2}$ of northern hemisphere data containing approximately 20.2 million point sources. Candidates were constrained to have detections at $J$ and $H$ bands with $J<16$ (2MASS signal-to-noise ratio $\sim 10$ limit), $J-H<0.3$ and $H-K_{s}<0.3,|b|>15^{\circ}$ (to eliminate source confusion in the plane), no minor planet correlations, and no optical counterparts (USNO-A; Monet et al. 1998) within 5". The adopted near-infrared colors select objects similar to or cooler than Gl 229B, while excluding the overwhelming number of main-sequence stars. Note that this may exclude $L / T$ transition objects $\left(0.6 \leq J-K_{s} \leq 2.1\right)$, although the temperature range over which $\mathrm{CH}_{4}$ absorption causes this transition may be quite small (Reid et al. 1999). $J$ - and $H$-band detections were required to exclude false sources, although a $K_{s}$-band detection was not

\footnotetext{
${ }^{11}$ The primary defining feature of spectral class $\mathrm{T}$ is the appearance of the 1.6 and $2.2 \mu \mathrm{m}$ overtone bands of $\mathrm{CH}_{4}$. While these objects have also been called "methane" dwarfs, we note that the $3.3 \mu \mathrm{m}$ fundamental $\mathrm{CH}_{4}$ band is predicted to appear at higher temperatures, i.e., perhaps among the latest type L dwarfs.

${ }^{12}$ See the Explanatory Supplement to the 2MASS Spring 1999 Incremental Data Release by R. M. Cutri et al., which is at http://www.ipac.caltech.edu/ 2mass/releases/spr99/doc/explsup.html.
} 
TABLE 1

2MASS T Dwarf Candidates: Imaging Results

\begin{tabular}{|c|c|c|c|c|c|c|c|}
\hline $\begin{array}{l}\text { Object }^{\mathrm{a}} \\
\text { (1) }\end{array}$ & $\begin{array}{l}\text { Sample } \\
\text { (2) }\end{array}$ & $\begin{array}{c}J \\
(3)\end{array}$ & $\begin{array}{l}H \\
(4)\end{array}$ & $\begin{array}{l}K_{s} \\
(5)\end{array}$ & $\begin{array}{c}\text { Date } \\
\text { (1999 UT) } \\
\text { (6) }\end{array}$ & $\begin{array}{l}\text { Instrument } \\
\text { (7) }\end{array}$ & $\begin{array}{c}\text { Confirmed } \\
\text { (8) }\end{array}$ \\
\hline 2MASSI J1007406+180601 & A & $15.75 \pm 0.06$ & $15.70 \pm 0.14$ & $15.77 \pm 0.24$ & May 27 & Keck NIRC & $\mathrm{No}^{\mathrm{b}}$ \\
\hline 2MASSI J1047539+212423 & A & $15.82 \pm 0.06$ & $15.79 \pm 0.12$ & $>16.29^{\mathrm{c}}$ & May 27 & Keck NIRC & Yes \\
\hline 2MASSI J1059440+183442 ... & A & $15.95 \pm 0.07$ & $15.83 \pm 0.15$ & $15.65 \pm 0.19$ & May 27 & Keck NIRC & $\mathrm{No}^{\mathrm{b}}$ \\
\hline 2MASSW J1217111-031113 $\ldots \ldots \ldots$ & B & $15.85 \pm 0.07$ & $15.79 \pm 0.12$ & $>15.91^{\mathrm{c}}$ & May 19 & USNO Tek $2 \mathrm{k}$ & Yes \\
\hline 2MASSW J1225543-273947 ...... & $\ldots$ & $15.23 \pm 0.05$ & $15.10 \pm 0.08$ & $15.06 \pm 0.15$ & May 27 & Keck NIRC & Yes \\
\hline 2MASSW J1237392+652615 $\ldots \ldots \ldots$ & $\mathrm{B}$ & $15.90 \pm 0.06$ & $15.87 \pm 0.13$ & $>15.90^{c}$ & May 19 & USNO Tek $2 \mathrm{k}$ & Yes \\
\hline 2MASSW J1346464-003150 ${ }^{\mathrm{d}} \ldots \ldots$ & B & $15.86 \pm 0.08$ & $16.05 \pm 0.21$ & $>15.75^{\mathrm{c}}$ & May 27 & Keck NIRC & Yes \\
\hline 2MASSW J2110230-285509 .. & B & $15.43 \pm 0.05$ & $15.31 \pm 0.08$ & $15.34 \pm 0.15$ & May 27 & Keck NIRC & $\mathrm{No}^{\mathrm{b}}$ \\
\hline 2MASSW J2142333-035556. & B & $15.86 \pm 0.06$ & $15.69 \pm 0.14$ & $15.73 \pm 0.24$ & May 27 & Keck NIRC & $\mathrm{No}^{\mathrm{b}}$ \\
\hline
\end{tabular}

\footnotetext{
${ }^{a}$ Source designations for 2MASS discoveries are given as "2MASSx Jhhmmss[.]s \pm ddmmss", where the " $x$ " prefix varies depending upon which catalog the object originates, in this case "I" for 1999 Spring Release Data and "W" for the survey's working database. The suffix conforms to IAU nomenclature convention and is the sexigesimal right ascension and declination at J2000 equinox.

${ }^{\mathrm{b}}$ Probable uncataloged asteroid.

${ }^{\mathrm{c}}$ Not detected at $K_{s}$ band; given magnitude is a $95 \%$ confidence magnitude lower (bright) limit based on the background flux.

${ }^{\mathrm{d}}$ This object was previously discovered by the Sloan Digital Sky Survey (Tsvetanov et al. 1999).
}

required because of decreased sensitivity at this wavelength ${ }^{13}$ (the 2MASS $99.9 \%$ completeness limit is $K_{s} \sim 14.3$ ). Nondetections on Palomar Observatory Sky Survey-I (POSS-I; Minkowski \& Abell 1963) are imposed because these objects have extremely red optical-infrared colors (Gl 229B $R-J \approx 9$; Golimowski et al. 1998).

The second set (sample B) was taken from $3420 \mathrm{deg}^{2}(\approx 28$ million point sources) of northern and southern hemisphere data with search criteria of $J-H<0.2$ and $H-K_{s}<0.2, J<16$, $|b|>20^{\circ}$, no minor planet correlations, and no POSS-I or POSS-II (Reid et al. 1991) detections. Again, sources were required to have $J$ - and $H$-band detections but were not required to have $K_{s}$-band detections.

${ }^{13} K_{s}$ nondetections are reported as limiting magnitudes (no object detected at given coordinates to $95 \%$ confidence level). In this case, the $H-K_{\text {s }}$ color limit is still required to be less than $0.3 \mathrm{mag}$.

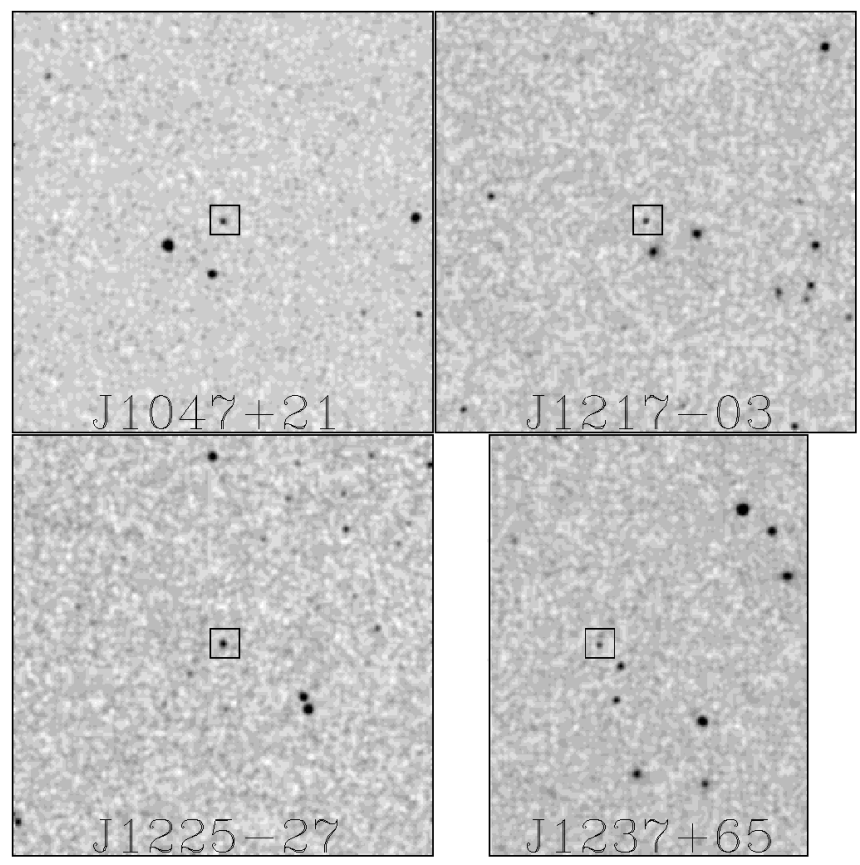

FIG. 1.- $J$-band images of the four new 2MASS T dwarfs: 2MASS $\mathrm{J} 1047+21$, 2MASS J1217-03, 2MASS J1225-27, and 2MASS J1237+65. Fields are $5^{\prime} \times 5^{\prime}$ (except for 2MASS J1237+65, which is $3{ }^{\prime} 8 \times 5^{\prime}$ ) with north up and east to the left. A $20^{\prime \prime} \times 20^{\prime \prime}$ box is drawn around each T dwarf.
The search criteria selected 349 candidates from sample A and 319 candidates from sample B. Subsequent inspection of POSS and 2MASS data to rule out faint optical sources (background stars) and proper motion stars eliminated all but 12 candidates from sample A and all but 11 from sample B. Three candidates from sample A and five from sample B were accessible for observation in May and are listed in Table 1, which gives the object name (col. [1]), sample (col. [2]), and 2MASS $J$-, $H$-, and $K_{s}$-band photometry (cols. [3]-[5]). The two samples are spatially distinct and can be considered complete in the region of sky observable during our investigation: $548 \mathrm{deg}^{2}$ in sample A and $1236 \mathrm{deg}^{2}$ in sample B.

One additional object, 2MASS J1225-27, which was previously identified as a T dwarf candidate (Burgasser et al. 1998) but is not a member of either sample, was also included in our follow-up and is listed in Table 1.

\subsection{Imaging Data}

The largest source of contamination among our $\mathrm{T}$ candidates is uncataloged minor planets (Burgasser et al. 1998; K99), which have blue near-infrared colors $\left(J-K_{s} \sim 0.3\right.$; Veeder et al. 1995; Sykes et al. 1999) and will not match a POSS-I or -II source because of motion. While catalogued minor planets are flagged by the 2MASS processing pipeline, uncataloged minor planets remain, and reimaging is required to eliminate them from the candidate pool.

Eighty minute $z^{\prime}$-band (Fukugita et al. 1997) exposures were obtained for two candidates, 2MASS J1217-03 and 2MASS $\mathrm{J} 1237+65$, using the USNO Flagstaff $1.55 \mathrm{~m}$ Tek2k Camera on 1999 May 19 (UT). The remaining targets in Table 1 were imaged at $K$ band using the Keck I Near-Infrared Camera (NIRC; Matthews \& Soifer 1994) on 1999 May 27 (UT). Results from these reimaging campaigns are summarized in Table 1, columns (6)-(8). In total, five of nine objects were confirmed at their expected positions. The 2MASS J-band images of the new confirmed candidates are shown in Figure 1. Designated names (col. [1]), 2MASS $J$-magnitudes and $J-H$, $H-K_{s}$, and $J-K_{s}$ colors (cols. [2]-[5]), and estimated distances (col. [6]) are listed in Table 2.

\section{SPECTROSCOPIC DATA}

An initial identifying optical (8000-11000 A) spectrum for 2MASS J1237+65 was obtained from the Palomar 200" Double Spectrograph (Oke \& Gunn 1982) on 1999 May 24 (Bur- 
TABLE 2

CONFIRMED 2MASS T DWARFS

\begin{tabular}{|c|c|c|c|c|c|}
\hline $\begin{array}{l}\text { Object } \\
\text { (1) }\end{array}$ & $\begin{array}{c}J \\
(2)\end{array}$ & $\begin{array}{c}J-H \\
(3)\end{array}$ & $\begin{array}{c}H-K_{s} \\
(4)\end{array}$ & $\begin{array}{c}J-K_{s} \\
\quad(5)\end{array}$ & $\begin{array}{c}\text { Estimated Distance }^{\mathrm{a}} \\
\text { (pc) } \\
(6)\end{array}$ \\
\hline 2MASSW J1217111-031113. & $15.85 \pm 0.07$ & $0.06 \pm 0.14$ & $<-0.12$ & $<-0.06$ & 14 \\
\hline 2MASSW J1346464-003150 ${ }^{\mathrm{b}} \ldots \ldots$ & $15.86 \pm 0.08$ & $-0.19 \pm 0.22$ & $<0.30$ & $<0.11$ & 14 \\
\hline 2MASSW J1225543-273947 ....... & $15.23 \pm 0.05$ & $0.13 \pm 0.10$ & $0.04 \pm 0.17$ & $0.17 \pm 0.16$ & 10 \\
\hline 2MASSI J1047539+212423 $\ldots \ldots \ldots$ & $15.82 \pm 0.06$ & $0.03 \pm 0.13$ & $<-0.50$ & $<-0.47$ & 13 \\
\hline 2MASSW J1237392+652615 ...... & $15.90 \pm 0.06$ & $0.03 \pm 0.14$ & $<-0.03$ & $<0.00$ & 14 \\
\hline
\end{tabular}

${ }^{a}$ Distance estimates assuming Gl 229B-like radii and $J$-band bolometric corrections and $T_{\text {eff }}=1000 \mathrm{~K}$.

${ }^{\mathrm{b}}$ This object was previously discovered by the Sloan Digital Sky Survey (Tsvetanov et al. 1999).

gasser et al. 1999). The data identify this object as very cool, since it is similar in appearance to the optical spectrum of Gl 229B (Oppenheimer et al. 1998), despite the exceedingly faint signal.

All confirmed candidates were then spectroscopically observed in the near-infrared using the Keck I NIRC grism on 1999 May 27-28 (UT). A 120 line $\mathrm{mm}^{-1}$ grism with the $H K$ order sorting filter was employed for first-order resolution $\lambda / \Delta \lambda=100$; the wavelength resolution was $60 \AA$ pixel $^{-1}$. Each target was imaged with NIRC in camera mode and then placed into either a 0".52 (for 2MASS J1217-03, 2MASS J1225-27, and 2MASS J1237+65) or 0 ".38 (for 2MASS J1047+21 and 2MASS J1346-00) wide slit. Total integration times of $1000 \mathrm{~s}$ were divided into five $200 \mathrm{~s}$ exposures with $5^{\prime \prime}$ on-slit dithers between exposures. The spectra were then pairwise subtracted to remove sky background, and each spectrum extracted separately. Flat-fielding was performed by obtaining a spectrum of a diffusely illuminated dome spot with identical instrumental settings. Each target spectrum was divided by a spectrum of a nearby SAO F star to correct for telluric absorption, and photometric standards were observed for flux calibrations. Individual exposures for each object were then averaged into a single, final spectrum.

Reduced spectra for all new 2MASS T dwarfs are shown in

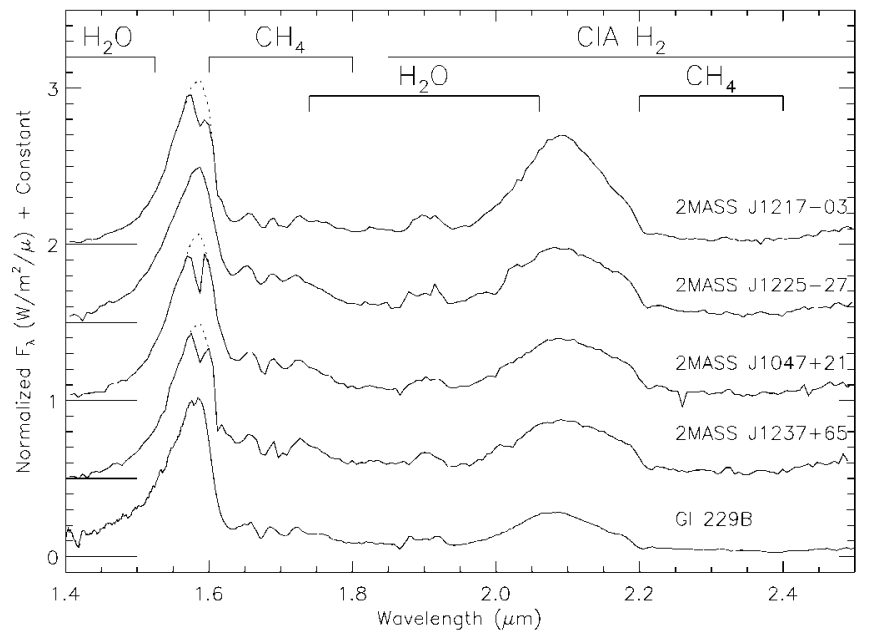

FIG. 2.-Near-infrared spectra (1.4-2.4 $\mu \mathrm{m})$ of the four new 2MASS T dwarfs, taken with the Keck I NIRC grism, along with Gl 229B spectral data from Leggett et al. (1999). The Gl 229B data has been smoothed to match the resolution of our NIRC observations. Objects are displayed from top to bottom in a preliminary temperature sequence: 2MASS J1217-03, 2MASS J1225-27, 2MASS J1047+21, 2MASS J1237+65, and G1 229B. Absorption bands for $\mathrm{H}_{2}, \mathrm{H}_{2} \mathrm{O}$, and $\mathrm{CH}_{4}$ are indicated. The "absorption features" seen at $1.58 \mu \mathrm{m}$ for all but 2MASS J1225-27 are due to saturation, and the dotted lines are extrapolations of the data in this region to fit to the 2MASS J1225-27 $H$-band peak. Spectra are normalized to their $H$-band peak.
Figure 2 along with Gl 229B ${ }^{14}$ (Leggett et al. 1999). All but one of the objects (2MASS J1225-27) were saturated at the $H$-band peak, resulting in a false "absorption" feature at $1.58 \mu \mathrm{m}$. The region of saturation was at most 3 pixels wide. An extrapolation of each saturated $H$-band peak using the 2MASS J1225-27 spectrum is indicated by a dotted line; each spectrum is normalized to this revised $H$-band peak. The 1.6 and $2.2 \mu \mathrm{m}$ methane bands are quite obvious in all of the confirmed candidates, identifying all as T dwarfs. Broadened $\mathrm{H}_{2} \mathrm{O}$ absorption bands shortward of 1.5 and $2.05 \mu \mathrm{m}$ are also evident, consistent with those found in Gl 229B (Oppenheimer et al. 1995). The $2.3 \mu \mathrm{m} \mathrm{CO}$ band seen in the L dwarfs (K99) is either overwhelmed by $\mathrm{CH}_{4}$ absorption or absent in the $\mathrm{T}$ dwarfs.

The spectra show significant variation in the ratio between the flux at 2.1 and $1.55 \mu \mathrm{m}$. As Figure 2 indicates, the $2.1 \mu \mathrm{m}$ peak flattens from 2MASS J1217-03 down to 2MASS $\mathrm{J} 1237+65$. In contrast, there is an apparent steepening in the core of the $1.6 \mu \mathrm{m} \mathrm{CH} \mathrm{CH}_{4}$ band, which is flat for 2MASS J1217-03 but decreases from 1.6 to $1.8 \mu \mathrm{m}$ for 2MASS J1225-27, 2MASS J1047+21, and 2MASS J1237+65.

\section{DISCUSSION}

\subsection{A Preliminary Sequence and $T_{\mathrm{eff}}$}

According to Burrows et al. (1997), the dominant form of molecular carbon switches from $\mathrm{CO}$ to $\mathrm{CH}_{4}$ at $T_{\text {eff }} \sim 1300 \mathrm{~K}$. $\mathrm{H}_{2} \mathrm{O}$ vapor bands, which have a profound influence on the nearinfrared spectra of M (Jones et al. 1994) and L (Delfosse et al. 1997) dwarfs, are also expected to be strong in T dwarfs. As Tokunaga \& Kobayashi (1999) have shown, $\mathrm{H}_{2}$ collisioninduced absorption (CIA) has a marked influence on the latest $\mathrm{L}$ dwarfs, noticeably depressing the spectrum in a broad region around $2.2 \mu \mathrm{m}$. This has also been seen in the reflectance spectrum of Jupiter (Danielson 1966). For the lower $T_{\text {eff }}$ 's of T dwarfs, $\mathrm{H}_{2}$ is expected to play a more dominant role.

Thus, for $T_{\text {eff }} \lesssim 1300 \mathrm{~K}$, the major absorbers in the nearinfrared $(J H K)$ spectra are $\mathrm{CH}_{4}, \mathrm{H}_{2} \mathrm{O}$, and $\mathrm{H}_{2}$. Since CIA affects the $K$ band more strongly than the $H$ band, particularly toward lower temperatures, the ratio of the flux at $H$ to that at $K$ should increase with cooler effective temperatures. This reasoning is the basis of our preliminary spectral sequence as displayed in Figure 2: 2MASS J1217-03, 2MASS J1225-27, 2MASS $\mathrm{J} 1047+21,2 \mathrm{MASS} \mathrm{J} 1237+65$, and Gl 229B. The $J$ band is even less influenced by CIA and little influenced by $\mathrm{CH}_{4}$, so that the same argument should also hold true for $J-K_{s}$; that is, we expect that cooler T dwarfs also have bluer $J-K_{s}$ colors. Four of the five objects were not detected at $K_{s}$ by 2MASS,

\footnotetext{
${ }^{14}$ The NIRC spectrum of the SDSS rediscovery, 2MASS J1346-00, will be presented in Burgasser et al. (1999).
} 
however, so these color relations require further observational investigation.

If we assume that these dwarfs have $8.5<\log$ (age, Gyr) $<$ 9.5 , typical of isolated field objects, then based on the observed colors and the assumption that all of the objects are warmer than Gl 229B, the 2MASS T dwarfs are likely to have $950<T_{\text {eff }}<1300 \mathrm{~K}$ (see Fig. 9 of Burrows et al. 1997). It must be stressed that this is a preliminary assessment of the temperatures of these objects, and no consideration for other spectral influences such as metallicity and gravity have been made. Better $T_{\text {eff }}$ estimates of these $\mathrm{T}$ dwarfs will require model fitting of their spectra from the far optical through the near-infrared. This analysis will be left to a future paper.

\subsection{Space Density and the Brown Dwarf Mass Function}

As indicated in Table 2, objects appear to lie within the $J-H<0.2$ and $H-K_{s}<0.2$ color region specified for sample B, even 2 MASS J1047+21, which was selected from a slightly broader color cut. If we assume that these colors are typical for all methane dwarfs, then we can make a preliminary assessment of the warm $\left(900<T_{\text {eff }}<1300 \mathrm{~K}\right) \mathrm{T}$ dwarf space density. With one detection in $548 \mathrm{deg}^{2}$ of sample A and three detections in $1236 \mathrm{deg}^{2}$ of sample B, we obtain a mean surface density of $0.0022 \mathrm{~T}$ dwarfs $\mathrm{deg}^{-2}$, or 90 warm $\mathrm{T}$ dwarfs observable over the entire sky with $J<16$. If we make the simplifying assumption that all four confirmed $\mathrm{T}$ dwarfs in our sample have the same luminosity as Gl 229B, then this $J$-band limit corresponds to a distance limit of $\sim 13 \mathrm{pc}$ and thus a space density of $\approx 0.01$ warm $\mathrm{T}$ dwarfs $\mathrm{pc}^{-3}$. This value should be considered an upper limit, since the warmer $\mathrm{T}$ dwarfs likely sample beyond $13 \mathrm{pc}$. It is nonetheless comparable to the L dwarf density of 0.007 dwarfs $\mathrm{pc}^{-3}$ computed by K99. Comparison with the simulations of Reid et al. (1999) suggests a mass function $d N / d M \propto M^{-1}$ in the T dwarf regime, compa- rable to the relation for local late $\mathrm{M}$ dwarf stars (Reid et al. 1999).

\section{SUMMARY}

We have identified four new $\mathrm{T}$ dwarfs and one previously discovered T dwarf in $1784 \mathrm{deg}^{2}$ of 2 MASS survey data. These objects all show the hallmark 1.6 and $2.2 \mu \mathrm{m} \mathrm{CH}_{4}$ absorption bands that are characteristic of objects with $T_{\text {eff }} \lesssim 1300 \mathrm{~K}$. They show some variation in their $H$ - to $K$-band flux ratios, likely due to the combined absorption of $\mathrm{CH}_{4}, \mathrm{H}_{2} \mathrm{O}$, and $\mathrm{H}_{2}$. This allows us to make a preliminary attempt at a $\mathrm{T}$ dwarf spectral sequence. We determine a proemial space density estimate of $\lesssim 0.01$ warm $\mathrm{T}$ dwarfs $\mathrm{pc}^{-3}$, which is comparable to the L dwarf density from K99.

A. J. B. would like to thank Tom Geballe and Sandy Leggett for the use of their recalibrated Gl 229B UKIRT spectrum, Ben Oppenheimer for his valuable comments, Albert Burgasser for consultation on a $\mathrm{T}$ dwarf search database, and especially the 2MASS staff and scientists for their support and for pointing their telescopes in the right directions. J. D. K. acknowledges Michael Strauss, Jill Knapp, and Xiaohui Fan for sharing news of their $\mathrm{T}$ dwarf discovery prior to publication. A. J. B., J. D. K., I. N. R., and J. L. acknowledge funding through a NASA/JPL grant to 2MASS Core Project science. A. J. B., J. D. K., R. M. C., and C. A. B. acknowledge the support of the Jet Propulsion Laboratory, California Institute of Technology, which is operated under contract with the National Aeronautics and Space Administration. This publication makes use of data from the Two Micron All-Sky Survey, which is a joint project of the University of Massachusetts and the Infrared Processing and Analysis Center, funded by the National Aeronautics and Space Administration and the National Science Foundation.

\section{REFERENCES}

Burgasser, A. J., et al. 1998, BAAS, 30, 1393

1999, in ASP Conf. Ser., From Giant Planets to Cool Stars, ed. C. A. Griffith \& M. Marley (San Francisco: ASP), in press

Burrows, A., et al. 1997, ApJ, 491, 856

Danielson, R. E. 1966, ApJ, 143, 949

Delfosse, X., et al. 1997, A\&A, 327, 25

Fukugita, M., Ichikawa, T., Gunn, J. E., Doi, M., Shimasaku, K., \& Schneider, D. P. 1997, AJ, 111, 1748

Geballe, T. R., Kulkarni, S. R., Woodward, C. E., \& Sloan, G. C. 1996, ApJ, 467,101

Golimowski, D. A., Burrows, C. S., Kulkarni, S. R., Oppenheimer, B. R., \& Brukardt, R. A. 1998, AJ, 115, 2579

Jones, H. R. A., Longmore, A. J., Jameson, R. F., \& Mountain, C. M. 1994, MNRAS, 267, 413

Kirkpatrick, J. D., et al. 1999, ApJ, 519, 802 (K99)

Leggett, S. K., et al. 1999, ApJ, submitted

Marcy, G. W., \& Butler, R. P. 1995, BAAS, 186, 3306

Marley, M. S., Saumon, D., Guillot, T., Freedman, R. S., Hubbard, W. B., Burrows, A., \& Lunine, J. I. 1996, Science, 272, 1919

Matthews, K., \& Soifer, B. T. 1994, in Infrared Astronomy with Arrays: The Next Generation, ed. I. McLean (Dordrecht: Kluwer), 239

Mayor, M., \& Queloz, D. 1995, Nature, 378, 355

Minkowski, R., \& Abell, G. O. 1963, in Stars and Stellar Systems, Vol. 3, Basic Astronomical Data, ed. K. Aa Strand (Chicago: Univ. Chicago Press), 481
Monet, D., et al. 1998, USNO-A2.0 Catalog (Flagstaff: USNO)

Nakajima, T., Oppenheimer, B. R., Kulkarni, S. R., Golimowski, D. A., Matthews, K., \& Durrance, S. T. 1995, Nature, 378, 463

Oke, J. B., \& Gunn, J. E. 1982, PASP, 94, 586

Oppenheimer, B. R., Kulkarni, S. R., Matthews, K., \& Nakajima, T. 1995, Science, 270, 1478

Oppenheimer, B. R., Kulkarni, S. R., Matthews, K., \& van Kerkwijk, M. H. 1998, ApJ, 502, 932

Rebolo, R., Zapatero-Osorio, M. R., \& Martín, E. 1995, Nature, 377, 129

Reid, I. N., et al. 1991, PASP, 103, 661 1999, ApJ, 521, 613

Ruiz, M. T., Leggett, S. K., \& Allard, F. 1997, ApJ, 491, L107

Skrutskie, M. F., et al. 1997, in The Impact of Large-Scale Near-IR Sky Surveys, ed. F. Garzon (Dordrecht: Kluwer), 25

Stauffer, J., Hamilton, D., Probst, R., Rieke, G., \& Mateo, M. 1989, ApJ, 344, 21

Strauss, M. A., et al. 1999, ApJ, 522, L61

Sykes, M. V., et al. 1999, Icarus, submitted

Tokunaga, A. T., \& Kobayashi, N. 1999, AJ, 117, 1010

Tsvetanov, Z. I., et al. 1999, ApJ, submitted

Veeder, G. J., Matson, D. L., Owensby, P. D., Gradie, J. C., Bell, J. F., \& Tedesco, E. F. 1995, Icarus, 114, 186

York, D., et al. 1999, in preparation 